Naturalismus

Manifeste und Dokumente zur deutschen

Literatur 1880-1900 
Naturalismus

\section{Manifeste und Dokumente zur deutschen Literatur 1880-1900}

Herausgegeben von Manfred Brauneck und Christine Müller

J. B. METZLERSCHE VERLAGSBUCHHANDLUNG STUTTGART 
CIP-Kurztitelaufnahme der Deutschen Bibliothek

Manifeste und Dokumente zur deutschen Literatur. Stuttgart : Metzler

1880 - 1900, Naturalismus / hrsg. von Manfred Brauneck u. Christine Müller. 1987.

ISBN 978-3-476-00602-8 kart.

ISBN 978-3-476-03230-0 (eBook)

DOI 10.1007/978-3-476-03230-0

ISBN 978-3-476-00601-1 geb.

NE: Brauneck, Manfred [Hrsg.]

Dieses Werk einschließlich aller seiner Teile ist urheberrechtlich geschützt. Jede Verwertung außerhalb der engen Grenzen des Urheberrechtsgesetzes ist ohne $\mathrm{Zu}$ stimmung des Verlags unzulässig und strafbar. Das gilt insbesondere für Vervielfältigungen, Übersetzungen, Mikroverfilmungen und die Einspeicherung und Verarbeitung in elektronischen Systemen.

(C) 1987 Springer-Verlag GmbH Deutschland Ursprünglich erschienen bei J.B. Metzlersche Verlagsbuchhandlung und Carl Ernst Poeschel Verlag GmbH in Stuttgart 1987 


\section{Inhalt}

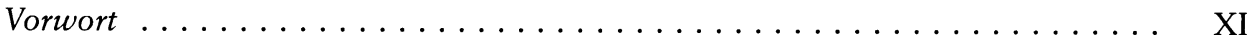

\section{Programmatik und Kunsttheorie}

a) Früheste Positionen

1 Heinrich Hart: Die Entwicklung der Künste (1877) . . . . . . . . . . . . . 3

2 Heinrich Hart: Neue Welt. Literarischer Essay $(1878) \quad \ldots \ldots \ldots \ldots$. . . . . . 7

b) Gruppenbildungen

Der »Hart-Kreis« (Berlin)

3 Heinrich Hart und Julius Hart: Graf Schack als Dichter (1883) . . . . . . . . . 19

4 Hermann Conradi: Unser Credo (1885) . . . . . . . . . . . . . . . . . 22

5 Ernst Henriet Lehnsmann: Die Kunst und der Sozialismus (1885) . . . . . . . . 23

6 Redaktion und Verlag der Zeitschrift "Die Gesellschaft«:

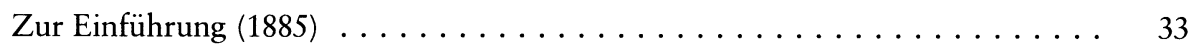

7 Julius Hillebrand: Naturalismus schlechtweg! (1886) . . . . . . . . . . . . 36

8 Karl Bleibtreu: Revolution der Litteratur $(1886,1887) \ldots \ldots \ldots$

9 Conrad Alberti: Die zwölf Artikel des Realismus. Ein litterarisches Glaubensbekenntnis (1889) . . . . . . . . . . . . . . . . . . . . . . 49

10 [Thesen der freien litterarischen Vereinigung »Durch!«] (1886) . . . . . . . . 58

11 Bruno Wille: [Protokoll der Sitzung des Vereins "Durch« vom 22. April 1887] . . 61

12 [Redaktion der Zeitschrift »Freie Bühne»]: Zum Beginn (1890) . . . . . . . . 63

13 Arno Holz: Zola als Theoretiker $(1890) \ldots \ldots \ldots \ldots$

14 Julius Röhr: Das Milieu in Kunst und Wissenschaft (1891) . . . . . . . . . 72

c) Grundlagen der Kunsttheorie

15 Hippolyte Taine: Philosophie der Kunst (1865, dt. 1902) _. . . . . . . . . . 78

16 Emile Zola: Der Experimentalroman $(1879$, dt. 1904) . . . . . . . . . . . 87 


\section{d) Kunsttheoretische Positionen}

17 Wilhelm Bölsche: Die naturwissenschaftlichen Grundlagen der Poesie. Prolego-

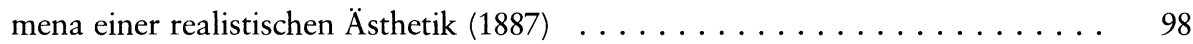

18 Max Kretzer: Objektivität und Subjektivität in der Dichtung (1889) . . . . . . . . 113

19 Heinrich Hart: Die realistische Bewegung. Ihr Ursprung, ihr Wesen,

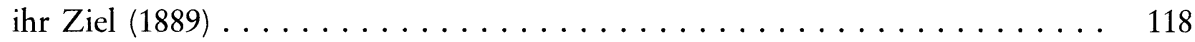

20 Julius Hart: Phantasie und Wirklichkeit. Eine Betrachtung auf Grund des Voß'schen Romans "Daniel, der Konvertit" (1889) . . . . . . . . . . . . . . . . . 129

21 Julius Hart: Der Kampf um die Form in der zeitgenössischen Dichtung. Ein Beitrag zugleich zum Verständniß des modernen Realismus (1890) . . . . . . 132

22 Arno Holz: Die Kunst. Ihr Wesen und ihre Gesetze (1891) . . . . . . . . . . . 140

23 Wilhelm Bölsche: Die sozialen Grundlagen der modernen Dichtung (1897) . . . 151

\section{e) Naturalismus und die Tendenzproblematik}

24 Heinrich Hart und Julius Hart: Friedrich Spielhagen und der deutsche Roman der Gegenwart (1884) . . . . . . . . . . . . . . . . . . . 162

25 Otto Ernst: Die Scheu vor der Tendenzdichtung (1890) . . . . . . . . . . . . 163

26 Hans Merian: Lumpe als Helden. Ein Beitrag zur modernen Ästhetik (1891) . . 168

\section{f) „Über den Naturalismus hinaus"}

27 Hermann Bahr: Die Krisis des Naturalismus $(1890,1891) \ldots \ldots \ldots \ldots$. . . . . . 180

28 Hermann Bahr: Die Überwindung des Naturalismus (1891) . . . . . . . . . . . 186

29 Leo Berg: Der Naturalismus. Zur Psychologie der modernen Kunst (1892) . . 190

\section{Die Gattungen}

\section{a) Der naturalistische Roman}

30 Heinrich Hart und Julius Hart: Friedrich Spielhagen und der deutsche Roman der Gegenwart $(1884) \ldots \ldots \ldots \ldots$. . . . . . . . . . . . . . . 203

31 Irma von Troll-Borostyani: Der französische Naturalismus (1886) . . . . . . . . 212

32 Franziska von Kapff-Essenther: Der Anfang vom Ende des Romans (1886) . . . 216

33 Karl Bleibtreu: "Größenwahn« $(1888) \ldots \ldots \ldots \ldots$. . . . . . . . . . . . . . 221

34 Wilhelm Bölsche: Goethes Wahlverwandtschaften im Lichte moderner

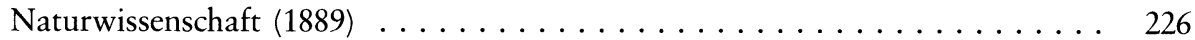

35 [Anonym]: Die Reichshauptstadt im Roman (1883) . . . . . . . . . . . . 235

36 Max Kretzer: Zur Entwicklung und Charakteristik des "Berliner Romans« (1885) 
37 W.[ilhelm] B.[los]: Meister Timpe. Sozialer Roman von Max Kretzer (1888) . . 245

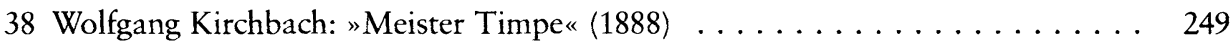

39 Wilhelm Bölsche: Die Poesie der Großstadt (1890) . . . . . . . . . . . . . . 253

\section{b) Drama und Theater}

Zeitgenössische Dramatik:

40 Heinrich Hart und Julius Hart: Das "Deutsche Theater" des Herrn L'Arronge

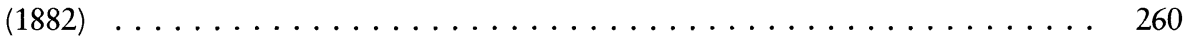

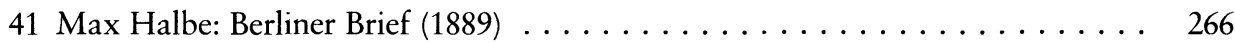

42 Otto Brahm: Der Naturalismus und das Theater $(1891) \ldots \ldots \ldots \ldots$. . . . . . 271

43 Otto Brahm: [...] Von alter und neuer Schauspielkunst (1892) . . . . . . . . 277

44 Paul Ernst: Die Anfänge des modernen Dramas (1897) . . . . . . . . . . . . 280

Naturalistische Dramaturgie und ihre Kritiker:

45 Gustav Schwarzkopf: Die Familie Selicke. Drama von Arno Holz und Johannes Schlaf $(1890) \ldots \ldots \ldots \ldots \ldots$. . . . . . . . . . . . . . . . . 284

46 Richard Dehmel: Die neue deutsche Alltagstragödie (1892) . . . . . . . . . . 289

47 Arno Holz: Evolution des Dramas $(1896,1925) \ldots \ldots \ldots 5$

48 Johannes Schlaf: Vom intimen Drama $(1898) \ldots \ldots \ldots \ldots$. . . . . . . . . . 298

49 Friedrich Spielhagen: Das Drama, die heutige litterarische Vormacht (1898) _ . 303

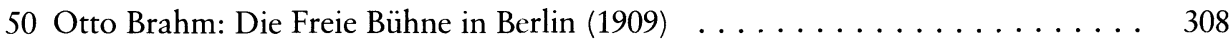

51 Heinrich Bulthaupt: Theater und Gesellschaft (1894) . . . . . . . . . . . . . . 319

52 Otto Brahm: Die Freie Volksbühne $(1890) \ldots \ldots \ldots \ldots \ldots$. . . . . . . . . 328

53 Richard Grelling: Die Maßregelung der Freien Volksbühne (1894) . . . . . . . . 333

54 Paul Schlenther: Theater und Umsturz (1895) . . . . . . . . . . . . . . . . . 339

c) Lyrik

55 Hermann Conradi: Unser Credo (1885) . . . . . . . . . . . . . . . 347

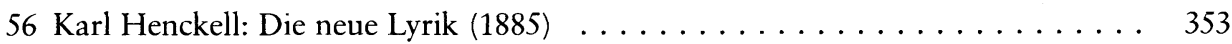

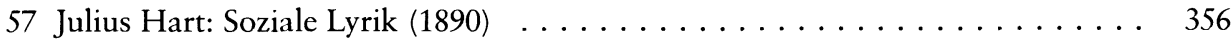

58 Arno Holz: Revolution der Lyrik $(1899) \ldots \ldots \ldots \ldots$. . . . . . . . . . . 364

\section{Zeitgenössische Rezeption und Kritik}

a) Naturalismus als Ausdruck »unserer Zeit»

59 Leopold von Sacher-Masoch: Die naturalistische Epidemie (1889) _ . . . . . . 375

60 Karl Frenzel: Der moderne Realismus (1891) _ . . . . . . . . . . . . . 380

61 Max Lorenz: Der Naturalismus und seine Überwindung (1899) . . . . . . . . . . 392 


\section{b) Abgrenzungen und Resümees}

62 Wolfgang Kirchbach: Realismus, Idealismus, Naturalismus in den gegenwärtigen europäischen Litteraturen $(1888)$. . . . . . . . . . . . . . . . . . . . . . 397

63 Maximilian Harden: Naturalismus (1890) . . . . . . . . . . . . . . . . . . . 403

64 Ernst von Wolzogen: Humor und Naturalismus (1890) . . . . . . . . . . . . . 407

65 Friedrich M. Fels: Naturalistische Literatur in Deutschland (1890) . . . . . . . . 415

66 Christian Ehrenfels: Wahrheit und Irrtum im Naturalismus (1891) . . . . . . . . 420

67 Die Zukunft der deutschen Litteratur im Urteil unserer Dichter und Denker. Eine Enquête von Kurt Grottewitz [Beiträge von Kurt Grottewitz, Julius Rodenberg, Heinrich Bulthaupt, Ernst Eckstein, Konrad Telmann, Gerhard von Amyntor, Karl von Perfall, Gerhart Hauptmann, Wolfgang Kirchbach, Karl Henckell, Ernst von Wolzogen, Maurice von Stern, Maria Janitschek, Hans Land, Max Nordau, Arno Holz, Johannes Schlaf, Franz Servaes, Ludwig Jacobowski, Eduard von

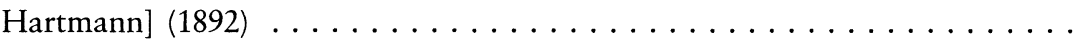

c) Deutschtum versus Internationalismus. Die Naturalismuskritik der Nationalkonservativen

68 Erwin Bauer: Die "Modernen" in Berlin und München (1891) . . . . . . . . . . . 445

69 [Fritz] L.[ienhard]: Nationale Ästhetik (1892) . . . . . . . . . . . . . . . . . . . . 453

\section{d) Rückblicke}

70 Hermann Bahr: Die Krisis des Naturalismus $(1890,1891)$. . . . . . . . . . . . 458

71 Hermann Bahr: Die Überwindung des Naturalismus (1891) . . . . . . . . . . . 458

72 Adolf Bartels: Die Alten und die Jungen. Ein Beitrag zur deutschen Litteraturgeschichte der Gegenwart (1896) . . . . . . . . . . . . . . . . . . 458

79 Samuel Lublinski: Ein Schulbeispiel des Naturalismus (1900) . . . . . . . . . . 466

\section{e) Naturalismus vor Gericht}

74 Conrad Alberti: Der Realismus vor Gericht (1890) . . . . . . . . . . . . . . .

75 »Im Namen des Königs . . .«[Urteil im Zensurprozeß betreffend das Schauspiel »Hanna Jagert" von Otto Erich Hartleben.] $(1892,1894)$. . . . . . . . . . . . . . 477

76 [Anonym]: Die Theaterzensur in Berlin (1893) . . . . . . . . . . . .

77 Richard Grelling: Glossen zum Weberprozeß. Mit einem Anhang: Censur-Prozeß betreffend $»$ Die Weber" von Gerhart Hauptmann (1894) . . . . . . . . . . . . 


\section{f) Naturalismus und Sozialdemokratie}

78 [Anonym]: Kunst und Volk $(1890) \ldots \ldots \ldots \ldots \ldots \ldots \ldots$

79 Paul Ernst: Die neueste literarische Richtung in Deutschland (1891) . . . . . . 512

80 W.[ilhelm] Liebknecht: Brief aus Berlin (1891) . . . . . . . . . . . . . . 524

81 W.[ilhelm] Liebknecht: Brief aus Berlin (1891) . . . . . . . . . . . . . . 526

82 Otto Brahm: Naturalismus und Sozialismus (1891) . . . . . . . . . . . . . 531

83 Michael Georg Conrad: Die Sozialdemokratie und die Moderne (1891) . . . . . 534

84 Robert Schweichel: Deutschlands jüngste Dichterschule (1891) _ . . . . . . . 540

85 Julius Hart: Ein sozialdemokratischer Angriff auf das »jüngste Deutschland «

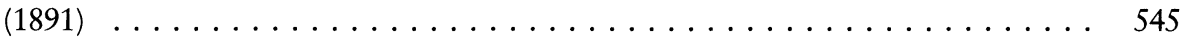

86 Franz Mehring: Etwas über den Naturalismus (1892) . . . . . . . . . . . . 550

87 Franz Mehring: Der heutige Naturalismus $(1892) \ldots \ldots \ldots \ldots$

88 Edgar Steiger: Das arbeitende Volk und die Kunst (1896) . . . . . . . . . . . 556

89 Edgar Steiger: Kunst und Sittlichkeit (1896) . . . . . . . . . . . . . . . . . . 559

90 Protokoll über die Verhandlungen des Parteitages der Sozialdemokratischen Partei Deutschlands. Abgehalten zu Gotha vom 11. bis 16. Oktober 1896 (Auszüge) .

91 Franz Mehring: Kunst und Proletariat (1896) . . . . . . . . . . . . . . . 578

92 Franz Mehring: Ästhetische Streifzüge $(1898 / 99)$. . . . . . . . . . . . . . . . . . 584

\section{g) Ibsen-Rezeption im Umfeld des deutschen Naturalismus}

93 Ludwig Fulda: Henrik Ibsen und das deutsche Drama (1886) . . . . . . . . . . 597

94 Otto Brahm: Henrik Ibsen's Gespenster in Berlin (1887) . . . . . . . . . . 605

95 Hermann Bahr: Henrik Ibsen $(1887,1890) \ldots \ldots \ldots \ldots$. . . . . . . . . . . . 609

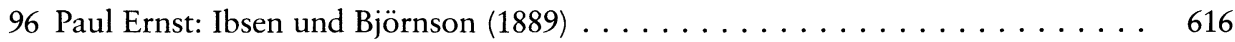

97 L[aura] Marholm: Die Frauen in der skandinavischen Dichtung (1890) . . . . . 624

98 Max Nordau: Der Ibsenismus (1893) _ . . . . . . . . . . . . . . . . . 627

99 Emil Reich: Ibsens Dramen (1894) _ . . . . . . . . . . . . . . . . . . 634

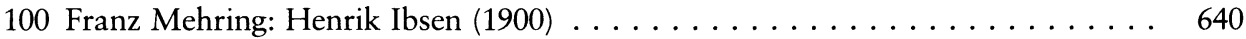

\section{b) Zola-Rezeption in Deutschland}

101 Ludwig Pfau: Emile Zola $(1880) \ldots \ldots$. . . . . . . . . . . . . . . . . . 646

102 Heinrich und Julius Hart: Für und gegen Zola (1882) _ . . . . . . . . . . . . 655

103 Gerhard von Amyntor: Zur Orientierung über den Zolaismus (1884) . . . . . . 660

104 Michael Georg Conrad: Zola und Daudet $(1880,1885) \ldots \ldots$. . . . . . . . 663

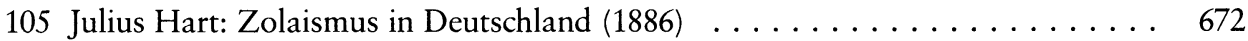

106 Fritz Mauthner: Daudet und Zola (1887) . . . . . . . . . . . . . . . . . . . . 679

107 Georg Brandes: Emile Zola (1888) _ . . . . . . . . . . . . . . . . . . . . 683 
108 Maximilian Harden: Zola und sein Menschenvieh (1890) . . . . . . . . . . . 702

109 Hermann Bahr: Emile Zola $(1890,1891) \ldots \ldots . . \ldots \ldots$. . . . . . . . . 709

110 Von einem Deutschen [= Julius Langbehn]: Rembrandt als Erzieher (1890) . . 713

111 Paul Lafargue: "Das Geld" von Zola (1892) . . . . . . . . . . . . . . . . . . . . 719

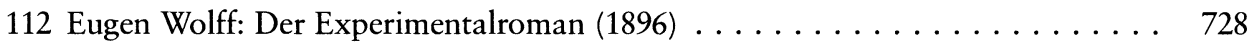

113 Franz Mehring: Emile Zola (1902) . . . . . . . . . . . . . . . . . . 732

i) Die Rezeption von Gerhart Hauptmanns Stück »Die Weber"

114 Wilhelm Bölsche: Gerhart Hauptmanns Webertragödie (1892) _ . . . . . . . 737

115 Otto Brahm: Hauptmann's Weber $(1892) \ldots \ldots \ldots \ldots \ldots \ldots$. . . . . . . 744

116 M.[aximilian] H.[arden]: Soziale Dramen $(1892) \ldots \ldots \ldots \ldots$. . . . . . . . 750

117 Fritz Mauthner: Freie Bühne. Die Weber (1893) _ . . . . . . . . . . . . . 755

118 [Anonym]: Die Weber. Schauspiel aus den vierziger Jahren von Gerhart Hauptmann. Verein Freie Bühne (1893) . . . . . . . . . . . . . . 760

119 Franz Mehring: Gerhart Hauptmanns "Weber« (1893) . . . . . . . . . . . . 763

120 Friedrich Spielhagen: Gerhart Hauptmanns »Die Weber" $(1893,1898)$. . . . . . 769

121 Theodor Fontane: Hauptmann. Die Weber $(1894,1898)$. . . . . . . . . . . . 774

122 John Schikowski: "Weber «-Premiere im Deutschen Theater in Berlin (1894) . . 775

123 Ludwig Zabel: Die Stellung des Staats und der Gebildeten zu Hauptmanns

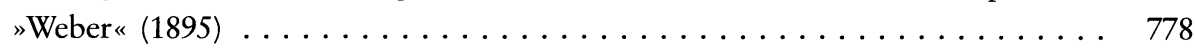

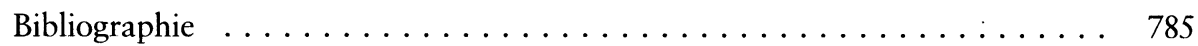

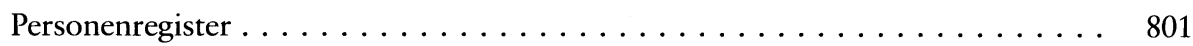

Verlagsregister . . . . . . . . . . . . . . . . . . . . 809 


\section{Vorwort}

»Da alle Naturgesetze, welche die mechanischen Vorgänge in der physischen Welt regeln, auch alle geistigen Vorgänge bestimmen, so ist auch die Kunst genau denselben Gesetzen unterworfen wie die mechanische Welt."

(Conrad Alberti: Glaubensbekenntnis)

"Als ob schon je ein Mensch irgendein Ding selbst reproduziert hätte und nicht bloß immer sein betreffendes Vorstellungsbild!«

(Arno Holz)

"Der Naturalismus an und für sich hat mit der Kunst nichts zu schaffen."

(Samuel Lublinski 1900 im Kunstwart)

Aus der Sicht des aktuellen literarischen Lebens gehört der Naturalismus ohne Zweifel zu jenen Erscheinungen der neueren deutschen Literatur, denen Leser eher mit frostiger Zurückhaltung gegenüberstehen. Kaum eine Erzählung, ein Roman, weniger noch die Lyrik des deutschen Naturalismus gehören zum Bestand historischer Werke, die dauerhaften Eingang in ein Literaturrepertoire gefunden haben, dessen stets neue Aneignung Element literarischer Kultur geworden ist. Allenfalls im Theater sind zwei, drei Stücke des jungen Gerhart Hauptmann gelegentlich zu sehen.

Reserviertheit kennzeichnet diese bemerkenswerte Rezeptionsgeschichte. Kunstlosigkeit, Trivialität und Tendenz - das sind die Stichworte unter denen ästhetische Naturalismuskritik sich von Anfang an weitgehend einig ist. So konstatiert auch Peter Bürger "eine beinahe einhellige Ablehnung " der Programmatik des Naturalismus, "und zwar von seiten von Autoren, die sehr verschiedene politische und literaturtheoretische Positionen vertreten «; und: „Es ist nicht schwierig, dieses Ärgernis ausfindig zu machen; es liegt in dem Versuch, Literatur der Wissenschaft anzunähern. Damit droht die Spezifizität der Literatur verlorenzugehen. «[1] Dieser Vorbehalt wird aus der Sicht postmoderner Aufklärungsskepsis allenfalls noch verschärft; erhebt doch gerade der Naturalismus für sich den Anspruch einer programmatischen Moderne par excellence. Wann schon wurde eine ästhetische Formel von der Eingängigkeit des Arno Holzschen Kunstgesetzes gefunden: »Kunst = Natur $-\mathrm{x}$ «.

Leichter im Umgang mit dem Naturalismus tun sich sozialistisch-materialistische Positionen der Literaturwissenschaft; immerhin läßt sich der Naturalismus für die Rekonstruktion realistischer Tradition - mit gewissen Einschränkungen freilich - beanspruchen[2]; auch stellt sich unter diesem Blickwinkel das Verhältnis von Kunst und Wissenschaft spannungsfreier dar.

Diese Sachverhalte gilt es zu überprüfen. Schreibt die Forschung hier Positionen zeitgenössischer Kritik oder naturalistischer Selbstdarstellung lediglich weiter? Hat sich dabei ein Bild verfestigt, das sich zu einseitig an Programmpunkte hält, die für einzelne Autoren oder 
Richtungen, für Teilpositionen also, kennzeichnend sein mögen, nicht aber für die ganze Bewegung? Wie geschlossen, wie einheitlich in Programmatik und literarischer Erscheinungsweise ist der deutsche Naturalismus überhaupt; liegen hier die Verhältnisse vielleicht ähnlich wie bei dem sich vom Naturalismus stets schroff abgrenzenden Expressionismus, für den die Forschung mehrfach schon die Aufgabe des verklammernden, die Divergenzen der Standpunkte aber zu sehr verwischenden Begriffsetiketts reklamiert hat?[3] Dieter Borchmeyer nennt den Naturalismus "eine ästhetische Grenzposition, die zwar fast alle bedeutenden Schriftsteller vor 1900 irgendwann einmal eingenommen oder gestreift haben, die jedoch keine eigentliche Epocher gebildet hat «[4].

Die vorliegende Dokumentation bietet Texte, in denen sich die ästhetische und weltanschauliche Programmatik des Naturalismus, die Vielfalt seiner Positionen, Richtungen und Gruppierungen, vor allem auch deren Wandlungen über eineinhalb Jahrzehnte hin manifestieren. Dabei werden auch wesentliche Paradigmen der zeitgenössischen Rezeption und Kritik einbezogen, da sich in diesen Diskussionsfeldern die Standpunkte in besonders pointierter Weise artikulieren.

Ziel dieser Textsammlung ist es, das bestehende Bild der naturalistischen Bewegung überprüfbar zu machen, neue Fragestellungen aufzuwerfen und eine umfassende Arbeitsgrundlage für Forschung und Lehre zu schaffen.

Einige Anmerkungen zur Verständigung über den historischen Rahmen und die theoretischen Grundannahmen des Naturalismus: In Deutschland sind die Naturalisten seit ca. 1883 in Berlin und nur wenig später auch in München als neue Autorengeneration in der Öffentlichkeit präsent, sie reagieren mit ihrer literarischen Produktion, insbesondere auch mit einer Fülle programmatischer Erklärungen auf die Probleme der sich entwickelnden Industriegesellschaft, vor allem auch auf die desolate geistige Situation des Kaiserreiches nach den Gründerzeitjahren. Wissenschaft und Sozialismus, Volk und Nation sind die großen Themen dieser Frühphase; "Revolution der Literatur" (1887), so lautet das weitgesteckte Ziel. Dennoch enttäuschen die künstlerischen Resultate dieser stürmischen Proklamationen aus heutiger Sicht, verbleiben sie zunächst doch ganz in den konventionellen Genres und Techniken des literarischen Schreibens. Erst Arno Holz geht Anfang der 90er Jahre neue Wege, aber da ist der Naturalismus fast schon wieder am Ende. Daß Kunst von ihrem schöpferischen Ausgang her frei sein müsse von jeglicher Tendenz, ist für keinen der deutschen Naturalisten eine Frage, die er anders beantworten würde als sein konservativster Kritiker, freilich divergieren die Begründungen. Wenn die zeitgenössische Rezeption hier dennoch ein anderes Bild bietet, dann resultiert das nicht zuletzt auch daraus, daß die Ablehnung der Tendenz bei den Naturalisten nicht gleichzeitig verbunden ist mit der Anerkennung der Kunst als einem zweckfreien Reich des Schönen, sondern daß sie, die Tendenz als kunstfeindlich ablehnend, dennoch die Trennung von Kunst und Leben, Kunst und Gesellschaft, Kunst und Volk zu überwinden suchen. Verbindendes Element sind die neuen Inhalte.

Abbildlichkeit vermittelt in der Ästhetik des Naturalismus das Verhältnis von Kunst und Natur, von Kunst und Leben. Freilich erfährt der Abbildbegriff im Verlauf der naturalisti- 
schen Bewegung äußerst variierende inhaltliche Bestimmungen, die von einem ausgeprägten Subjektivismus bis zu einem positivistischen Objektivismus die verschiedensten Positionen umfassen. Arno Holz und Johannes Schlaf sind um Reproduktion der Alltagsrealität bemüht, die rigoros einer positivistischen Realitätsauffassung folgt; ihren Sekundenstilexperimenten ist aus anderen Epochen nichts Vergleichbares an die Seite zu stellen. Die Begründung von Holz' Kunsttheorie erfolgt unter Zuhilfenahme eines umfassenden, von den Naturwissenschaften adaptierten Gesetzesbegriffs. Kunst und Leben unterliegen aus dieser Sicht einem gleichen Determinationszusammenhang, der als Konstruktionsprinzip naturalistischer Wirklichkeitsgestaltung deren Wahrheitsgehalt garantieren soll. Auf eine "Soziologie der Kunst" (Arno Holz) hin entwickelt sich deswegen auch die naturalistische Kunsttheorie in Deutschland. Sie ist in ihren Konstitutionsprinzipien nicht weniger normativ als die mit Vehemenz verworfene idealistische Tradition, sie verlagert lediglich den Begründungszusammenhang ästhetischer Normativität, die freilich dem historischen Wandel überantwortet, damit aber nicht aufgehoben ist. Mit einem emphatisch proklamierten Wahrheitsbegriff, wie er etwa in Michael Georg Conrads Eröffnungserklärung für seine Zeitschrift Die Gesellschaft (1885) aber auch in zahllosen anderen Schriften der naturalistischen Essayistik zu finden ist, wird diesem Programm nachdrücklich moralische Legitimation verschafft. Der Wahrheitsbegriff verklammert Kunst und Leben, in ihm fallen die ethischen, erkenntniskritischen und ästhetischen Postulate des Naturalismus zusammen. Naturalistische Kunst spiegelt zwar nicht mehr das Schöne und Gute wider, jedoch Wahrheit und Wirklichkeit, kurzum: "modernes Leben «. Und darum ging es vor allem. Es sollten die Probleme der Gegenwart mit angemessenen Methoden der Darstellung und der Bewertung Eingang in die Kunst finden, anstelle idyllischer oder historistischer Daseinsverklärung und bloßer Formspielerei.

War die Frühphase des Naturalismus, Roman und Lyrik vor allem, kaum über die Vorgaben der literarischen Konvention, in Erzählweise und in der lyrischen Formensprache, hinausgekommen, wird um 1890 der Zugriff auf die neuen Stoffe freier, erhält naturalistisches Schreiben eigene Kontur, vor allem im Drama. Wo hier die Darstellung an die Grenzen der konventionellen Abbildverfahren und deren poetologischer Matrizen gerät, reagieren die Naturalisten nun mit der Auflösung gattungsgebundener Sprach- und Dramaturgiestrukturen. Unter dem Begriff der Episierung des Dramatischen werden diese Entwicklungen subsumiert, von der Kritik zunächst noch als Regelverstoß geahndet. Die Geschlossenheit der Werkform als strukturaler Entsprechung der Autonomie des Kunstwerks ist jedoch die verbindliche Grenze jedweden naturalistischen Formexperiments. Das Drama mit dem offenen Schluß, "endlos wie die Natur", bleibt ein Gedanke, der nicht weiterverfolgt wird; seine Stücke beendet Gerhart Hauptmann streng der Regel entsprechend. Konstruktionsprinzipien, die den mimetischen Rahmen sprengen würden, bleiben unter den Bedingungen naturalistischer Kunsttheorie ausgeschlossen. So erweist sich der Naturalismus letzendlich als das, was er in seiner Programmatik auch stets zu sein vorgab: "konsequenter Realismus « - in der Folge und als Endpunkt der Realismusentwicklungen des 19. Jahrhunderts.

Damit ist der kunsttheoretische Ort des Naturalismus umschrieben, zugleich aber auch 
seine Begrenztheit als ästhetisches Verfahren erkennbar. Nur in Ansätzen gelingt es dem Naturalismus, für die komplexen Umbruchsprozesse, denen die soziale Wirklichkeit und die weltanschaulichen Orientierungen in den Jahrzehnten des ausgehenden 19. Jahrhunderts unterworfen sind, eine künstlerische Form zu finden, die über die Bedingtheiten dieser Zeit hinausweist.

In der zeitgenössischen Rezeption steht die naturalistische Literatur in den aktuellen Widersprüchen der gesellschaftlichen Situation und wird daraus auch bewertet. Dabei ist zumindest von zwei Gegebenheiten auszugehen: von dem Engagement vieler Naturalisten für den Sozialismus und die politische Arbeiterbewegung, Anfang der 90er Jahre in der spannungsreichen Rolle einer Linksopposition gegenüber der sozialdemokratischen Parteiführung; und - von der politischen Bewertung naturalistischer Literatur durch die konservative Literaturkritik und durch die Ordnungsbehörden, letztere insbesondere im Zusammenhang von Zensurverfahren. Ihnen gilt der Naturalismus als internationalistisch, sozialistisch, schließlich gar als anarchistisch. In diesen Bewertungen schlägt die Dynamik der zeitgeschichtlichen politischen und weltanschaulichen Auseinandersetzungen in aller Schärfe in den Kulturbetrieb durch. Daß davon in erster Linie der Naturalismus betroffen war, ist wohl mehr ein Beweis für die Irritation, die diese Literatur für die Zeitgenossen offenbar darstellte, als für die eher halbherzigen Bekenntnisse naturalistischer Autoren zu den politischen Zielen der "Umsturzpartei«, als welche die Sozialdemokratie den Konservativen in jenen Jahren erschien. Die nachdrückliche Distanzierung der Sozialdemokratie vom Naturalismus entlastete diesen keineswegs gegenüber dem konservativen Verdikt. Ein weiterer Aspekt kommt hinzu; er betrifft das Theater, das um 1890 zur wichtigsten und öffentlich wirksamsten Vermittlungsinstitution naturalistischer Literatur wird. Die Freie Bühne und zeitweilig auch die Freie Volksbühne sind im Berlin der frühen 90er Jahre, dem Brennpunkt aller geistigen und politischen Auseinandersetzungen der Zeit, die exponiertesten Neuerungen im Kulturbetrieb. Sie sind es durch ihre Kritik am bestehenden Theaterwesen, die ihren Ausdruck gerade im Engagement für den deutschen und außerdeutschen Naturalismus findet, in der Durchsetzung neuer Stücke und Autoren, einer naturalistischen Schauspielkunst und Dramaturgie (Otto Brahm); und sie sind es vor allem auch auf Grund des Umgehens der geltenden Zensurverordnungen, wozu sie ihr Status als private Vereine in die Lage versetzt. Bei der Freien Volksbühne kommt eine politisch motivierte Veränderung der Distributions- und Rezeptionspraktiken des Theaters hinzu. Für eine kurze Zeit wird im Kontext dieser neuen Vermittlungsstrukturen eine Art proletarische Gegenöffentlichkeit organisiert. Die neue Literatur wird einem Arbeiterpublikum nahegebracht; die politische Dimension, die den Stücken an sich fehlt, wird durch die Produktions- und Rezeptionsstrukturen der Aufführung hergestellt. Tendenziell ist damit ein Bruch mit der bürgerlichen Auffassung von Kunst als einer zweckfreien Sphäre vollzogen. Wenn sich in diesem Zusammenhang aber auch darüberhinaus zeitweilig und auf wenige Werke bezogen eine politische Diskussion naturalistischer Literatur ergibt, begründet in der für den autoritären Staat charakteristischen Verschränkung von Politik, Kunst und Moral, so ist dies freilich ein Vorgang, der in der ästhetischen Theorie des Naturalismus kaum einen 
Rückhalt findet, sondern seine Bedingungen insbesondere der Dialektik der Rezeption verdankt. [5]

Der Aufbau der Dokumentation erfolgt in drei großen Kapiteln: Programmatik und Kunsttheorie(I), die Gattungen Roman, Drama und Theater, Lyrik(II), zeitgenössische Rezeption und Kritik(III). Ein Kapitel "Literarisches Leben" wurde nicht eingerichtet, da für Bereiche wie Verlagswesen, Bibliotheken, Leseverhalten, Situation der Schriftsteller etc. spezifische, nur für den Naturalismus charakteristische Gegebenheiten bislang von der Forschung nur sporadisch untersucht wurden. Der heutige Forschungsstand, an dem sich Dokumentationen wie die vorliegende letztendlich orientieren müssen, läßt eine umfassende Darstellung dieses Bereichs noch nicht zu. Hier liegen offenbar die größten Defizite in der Erforschung dieser Periode. Selbst Einrichtungen wie die Freie Bühne oder die Freie Volksbühne, deren Bedeutung für den Naturalismus offenkundig ist, können in diesem Zusammenhang nur begrenzt beansprucht werden, da deren Repertoiregestaltung keineswegs nur auf die naturalistische Theaterliteratur festgelegt war. Das Kapitel Rezeption bringt allerdings eine Reihe von Texten, die für diese Problematik einschlägig sind: Dokumente zu den Zensurverfahren, zur Literaturdebatte innerhalb der Sozialdemokratie, Charakterisierung von Lesergruppen im Zusammenhang literarischer Kritik u.a.

Der vorliegende Band war ursprünglich als Überarbeitung und zweite Auflage der von Erich Ruprecht 1962 herausgegebenen Textsammlung Literarische Manifeste des Naturalismus 1880-1892 geplant. Die Neukonzeption der gesamten Reihe der Manifeste führte dann jedoch zu einer völligen Neugestaltung auch des Naturalismus-Bandes, zu neuen inhaltlichen Schwerpunktsetzungen, einer neuen Form der Textkommentierung, vor allem auch einer beträchtlichen Umfangserweiterung.

Ein Bestreben bei der Textauswahl war es, die Dokumente in ihrer Argumentationsstruktur möglichst zu erhalten, sie nicht auf einschlägige "Kernstellen" zu verkürzen. Wo es vertretbar erschien, wurden einzelne Texte auch ungekürzt wiedergegeben.

Bei diesem Vorgehen wurde in Kauf genommen, daß gelegentlich Zuordnungsprobleme gegenüber der Gliederung der Dokumentation entstanden. In jedem der Texte steht zwar die Auseinandersetzung mit einer Thematik eindeutig im Mittelpunkt, dies bestimmt dann auch den Standort des Dokuments in der Gliederungssystematik; oft aber werden im gleichen Text auch Probleme mitangesprochen, die in der Systematik des Bandes an anderer Stelle aufgenommen werden. So ließen sich manche Beiträge mit guten Gründen auch unter anderen Gliederungstiteln subsumieren; z. B. finden sich in Texten zur Romantheorie (Kapitel II) oder in Rezeptionsdokumenten (Kapitel III) gelegentlich längere Passagen, die allgemeine Probleme der Kunsttheorie (Kapitel I) erörtern. Der Leser ist hier angewiesen, "quer « zu lesen; 
der Kommentar ist dabei behilflich und bietet die entsprechenden Verweisungen. Innerhalb der Kapitel und Abschnitte sind die Texte in der Regel chronologisch geordnet.

In einem ersten Abschnitt des Theoriekapitels (I) werden früheste Positionen dokumentiert, in denen sich Neuorientierungen einer literarischen Opposition Anfang der 80er Jahre abzeichnen. Entsprechend ihrer herausragenden Rolle in diesem Zusammenhang stehen hier die Beiträge der Brüder Hart im Mittelpunkt. Durch Gründungen von Zeitschriften und ihre maßgebliche Mitarbeit in den in diesen Jahren wie Pilze aus dem Boden schießenden literarischen Clubs und Vereinen nehmen sie eine Schlüsselstellung im Berliner Literaturbetrieb ein; ihre publizistische Ausstrahlung reicht jedoch weit über diesen lokalen Bereich hinaus. In den Beiträgen von Heinrich und Julius Hart zeichnet sich noch vor der Ausformulierung eines dezidiert naturalistischen Literaturprogramms das Konzept einer literarischen Moderne ab. Dabei geht es um die Begründung einer Reform des gesamten literarischen und weiter gefaßt auch des kulturellen Lebens in Deutschland, getragen vom Pathos nationalen Erneuerungswillens und unter Inanspruchnahme der Naturwissenschaften als Grundlage einer neuen, kritisch verstandenen und als realistisch propagierten Kunstauffassung: Nationalismus und Naturwissenschaft als Gegengift zur "Literatur der Salons«. In diesem auf das Nationale und eine neue Moralität verpflichteten Literaturprogramm erscheint das "Volk«, ganz im Sinne romantisch-nationaler Auffassung, als Träger und Garant der neuen Richtung. Nur wenige Jahre später wird dieses Programm von den Harts, in deutlicher Abgrenzung zu dem inzwischen etablierten Naturalismus, in die Forderung nach einer »neuen Klassik« weitergeführt.

In der Phase des Frühnaturalismus sind inhaltliche Verflachung, formales Epigonentum und Dilettantismus die erklärten gegnerischen Positionen, denen gegenüber eine Literaturbewegung der "nationalen Wiedergeburt « initiiert wird. Neben der Naturwissenschaft sind der Sozialismus und die Arbeiterbewegung die Fundamente, auf die der neue "realistische« Geist sich gründet. Der Beitrag von Ernst Lehmann, Die Kunst und der Sozialismus, der in diesen Zusammenhang gehört, markiert jenen letzteren Aspekt in aller Deutlichkeit und eröffnet die theoretische Diskussion über den die naturalistische Kunstprogrammatik durchgehend - und in den unterschiedlichsten Facettierungen schillernd - begleitenden Reflexionszusammenhang zwischen naturalistischer Literatur und Arbeiterbewegung (soziale Frage, Sozialismus, Sozialdemokratie, Anarchismus).

Aus diesem Umfeld bilden sich bald erste Gruppierungen heraus, in denen die neue Bewegung Kontur, vor allem auch ein bestimmteres Programm erhält. Neben dem Hart-Kreis und der ebenfalls Berliner literarischen Vereinigung "Durch!« (seit 1886) sind es vor allem die beiden Gruppen Münchner bzw. Berliner Literaten, versammelt um die Zeitschriften Die Gesellschaft (seit 1885) und Die Freie Bühne (seit 1889), die in der Dokumentation vorgestellt werden. In München ist es der Kreis um Michael Georg Conrad, dem Herausgeber der Gesellschaft. Programmatisch verschärft sich hier der allgemeine kulturkritische Gestus der neuen Bewegung, verbunden mit einer vehement zur Schau gestellten Antibürgerlichkeit. "Wahrheit" wird das Schlagwort der Münchner Gruppe, das bekennerhaft in dem Programmessay der Zeitschrift proklamiert wird. Wahrheitssinn als kulturkritische Haltung und Realis- 
mus werden zu Synonymen. Hinzu kommt, für die Münchner charakteristisch, ein romantizistischer Geniekult, der wesentlich von Conrads Wagner-Begeisterung und einer allgemeinen Nietzsche-Verehrung geprägt ist. Entscheidende Impulse für die Formulierung der naturalistischen Literaturprogrammatik gewinnen die Münchner vor allem aus der Rezeption des Werkes von Emile Zola. Allerdings wird Zolas Konzeption einer Angleichung von Kunst und Wissenschaft auch hier nicht angenommen, sondern findet, wie die Beiträge von Julius Hillebrand, Carl Bleibtreu und Conrad Alberti zeigen, bestenfalls im Sinne eines naturwissenschaftlich fundierten Realismus Eingang in die eigenen Positionsbestimmungen. Dabei werden erste Naturalismusdefinitionen formuliert; das neue Kunstprinzip erhält Systematik in verbindlich erklärten Grundsätzen (Conrad Albertis Glaubensbekenntnis). Ausgeführt wird die Grundlegung des naturalistischen Verfahrens in den modernen Naturwissenschaften und in der Auseinandersetzung mit der sozialen Frage, als den beiden »Zeichen der Zeit«. Zugleich entwickelt sich eine Gegensätzlichkeit der Lager bis hin zur polemischen Attacke: München versus Berlin. 1889 wird dort der Theaterverein "Freie Bühne " gegründet, 1890 erscheint die gleichnamige Zeitschrift, die bald Die Gesellschaft als programmatisches publizistisches Forum des Naturalismus ablöst und die Theoriediskussion in eine neue Phase überführt. Otto Brahm setzt gegenüber den Münchnern neue Akzente durch Anknüpfung an frühnaturalistische Positionen. Der künstlerische Subjektivismus wird zum unverzichtbaren Bestandteil naturalistischer Programmatik erklärt und gegen den konsequenten Objektivismus naturwissenschaftlicher Prägung abgegrenzt. Auch die Abgrenzung gegenüber Zolas Kunsttheorie wird fortgesetzt, insbesondere von Arno Holz. Insgesamt - und das ist die entscheidende Nuance der Berliner gegenüber dem Münchner Kreis, wird die Wissenschaftsorientierung von der Naturwissenschaft auf die Sozialwissenschaften erweitert, später zur Psychologie und zu lebensphilosophischen Positionen hin.

Eine zentrale Stellung nehmen in diesem ersten Teil der Dokumentation die Kapitel "Grundlagen der Kunsttheorie" und »Kunsttheoretische Positionen « ein. Neben den für die gesamte Naturalismusbewegung als Grundlagen geltenden Texten von Hippolyte Taine und Emile Zola stehen Bölsches Prolegomena als Versuch einer naturwissenschaftlichen Fundierung der Poesie auf der Basis eines idealistischen Monismus sowie der bisher von der Forschung kaum beachtete spätere Aufsatz Die sozialen Grundlagen der modernen Dichtung, dazu Texte von Heinrich und Julius Hart und vor allem von Arno Holz im Mittelpunkt.

Taine war der erste, der Darwins Idee einer durchgängigen Gesetzmäßigkeit natürlicher Entwicklungsprozesse auf das Gebiet der Geschichte der Literatur- und Kunstwissenschaft übertrug und über die Adaptionen einer aus den Naturwissenschaften übernommenen, monistisch-mechanistischen Methodologie diese Wissenschaften auf eine neue, "moderne " Grundlage zu stellen versprach. Alle Phänomene von Geschichte, Kunst und Literatur schienen Taine verursacht durch eine Trias von Bedingungsfaktoren, die er mit "race«, "milieu « und "moment « zusammenfaßte. Diesen Bedingungsfaktoren nachzuspüren und sie in möglichst lückenloser Kette zu rekonstruieren, sei alleinige Aufgabe der Wissenschaften: "In diesem Verstande verdammt die Wissenschaft weder noch begnadigt sie - sie stellt fest und erklärt «. 
Damit war der Positivismus in der Geschichtswissenschaft und in den Kulturwissenschaften grundgelegt. Emile Zola greift dieses Tainesche Konzept der Übertragung naturwissenschaftlicher Methodologie und Erkenntnisziele auf die Geschichtswissenschaft auf und systematisiert es in enger Anlehnung an die Experimenttheorie des Medizintheoretikers Claude Bernard zu seiner Theorie des Experimentalromans, einer weit über die Romantheorie hinausgehenden, auf die Gleichsetzung von Kunst und Wissenschaft zielenden kunsttheoretischen Programmatik. Zolas Schrift stand im Mittelpunkt der naturalistischen Theoriediskussion, sowohl bei den enthusiastischen Zola-Anhängern der Gesellschaft als auch bei den zunehmend auf Distanz zu Zola insistierenden Berliner Naturalisten. Die zentralen Problemstellungen naturalistischer Literaturtheorie sind in dieser Schrift vorgegeben. Die Beiträge von Bölsche, Max Kretzer, den Harts und Arno Holz markieren die Spielräume der ästhetischen Grundlagendiskussion im deutschen Naturalismus. Dabei ist Wilhelm Bölsches Entwicklung von der Schrift über die Naturwissenschaftlichen Grundlagen der Poesie (1887) hin zu dem zehn Jahre später vorgetragenen Konzept der Sozialen Grundlagen der modernen Dichtung (1897) symptomatisch für die Gesamtbewegung. Die Entwicklung verläuft von einer zunächst rigoros auf die Naturwissenschaften bezogenen Theorieorientierung ("Die Basis unseres gesamten modernen Denkens bilden die Naturwissenschaften«) zu einer Ausweitung des kunsttheoretischen Reflexionshorizonts (»Endlos lange vernachlässigt, treten heute zum ersten Mal die sozialen, die Massenbewegungen in der Geschichte hervor, das ,Volk، tritt allenthalben in Aktion «). Die Diskussion wird in der Spanne dieser Entwicklung zunehmend über den produktionsästhetischen Aspekt (Abbildproblematik) hinaus auf die Komplexität des bürgerlichen Kulturbetriebs als dem letztlich bestimmenden Rahmen literarischer Produktion und Rezeption bezogen. Das in dieser Verschiebung des Theorienansatzes angelegte Moment einer Politisierung der ästhetischen Diskussion wird freilich - entsprechend der Lagerbildung innerhalb der Sozialdemokratie - von Bölsche im Sinne des revisionistischen Flügels der Partei gelöst. Die Gegenposition, am markantesten vertreten durch Franz Mehring, ist zu diesem Zeitpunkt längst zu einer dezidiert politischen Kritik des Naturalismus gekommen. Diesen Zusammenhängen ist ein eigenes Kapitel (III,f) innerhalb dieser Dokumentation gewidmet.

In den Schriften von Heinrich und Julius Hart wird deutlich, in welchem Maße die Theoriediskussion im Umfeld des Naturalismus von Anfang an durch traditionalistische Vorstellungen mitbestimmt ist, die bei allem Neuerungswillen weniger auf einen konsequenten Neuansatz hinarbeiten, als vielmehr eine Reform der Literatur und des literarischen Lebens auf der Grundlage letzthin idealistischer Postulate anstreben: "Ideal-Realismus " heißt das Konzept der Harts in den 80er Jahren. Gerade in der Gegenüberstellung der von den Harts einerseits und Holz andererseits vertretenen Positionen ist erkennbar, welche Spannweite diese Diskussion umfaßt, aber auch welche konzeptionellen Gegensätze, ja Widersprüche unter der Bezeichnung Naturalismus hier subsumiert sind. So wird der Naturalismus als ein Prozeß erkennbar, in dessen Mittelpunkt die Auseinandersetzung um die Neugewinnung einer kritisch-eingreifenden, dem modernen Wissensstand adäquaten realistischen Ästhetik 
steht, bis von Arno Holz schießlich die Position eines ästhetizistischen Formalismus erreicht wird, der zugleich auch den Übergang in einen neuen Abschnitt des kunsttheoretischen Diskurses markiert.

"Kunst $=$ Natur $-\mathrm{x}$ «, diese handliche Formel, von Arno Holz geprägt, ist, bei aller Mißverständlichkeit, die sie birgt, als Manifestation naturalistischer Kunstauffassung in den allgemeinen Wissensstand über den Naturalismus eingegangen. Wie sehr damit aber nur ein Aspekt dieses Diskussionsspektrums repräsentiert und diese Position im ganzen der naturalistischen Literaturdebatte doch relativiert wird, wird aus der Gegenüberstellung mit den Texten der anderen Autoren deutlich. Damit wird freilich die überragende Bedeutung von Arno Holz als Theoretiker des deutschen Naturalismus keineswegs in Frage gestellt.

In einem eigenen Kapitel ausgegrenzt ist die im Zusammenhang mit der Naturalismusrezeption in Deutschland erneut aufgekommene Tendenzdiskussion. Dieser von der konservativen Naturalismuskritik eingeführte Aspekt stellt insofern für die Naturalisten eine besondere Herausforderung dar, als durch den Tendenzvorwurf der Objektivitätsanspruch des naturalistischen Programms grundlegend in Frage gestellt ist. Die Stellungnahmen zu dieser Frage, die wesentlich auch die Rezeptionsauseinandersetzungen mit dem Naturalismus bestimmen, erhellen einmal mehr die Gegensätzlichkeiten und Widersprüche der Standpunkte innerhalb der Naturalisten selbst wie im Lager ihrer Kritiker. Außer in den in dieses Kapitel aufgenommenen Texten der Brüder Hart, Otto Ernsts und Hans Merians ist die Auseinandersetzung mit dieser Frage ein durchgängiges Problem auch in den Beiträgen von Leo Berg, Wilhelm Bölsche u. a. Der Kommentar erläutert diese Bezüge.

"Über den Naturalismus hinaus« ist das letzte Kapitel des ersten Teils überschrieben. Die Texte von Hermann Bahr und Leo Berg bezeichnen Positionen der Erweiterung des naturalistischen Programms, die bereits in neue Richtungen weisen, generell bestimmt durch den Abbau der objektivistischen Aspekte der Kunsttheorie und die Vorbereitung ihrer Subjektivierung. Psychologisierung der Kunst tritt an die Stelle ihrer positivistisch-mechanistischen bzw. soziologischen Grundlegung.

Der zweite Teil der Dokumentation (II) bringt Texte, die die Diskussion der literarischen Gattungen unter den Prämissen naturalistischer Kunstauffassung belegen. Einige der Positionen sind vom Standpunkt der Rezeption bzw. der Naturalismuskritik aus entwickelt. Dabei sind die Gewichtungen durchaus unterschiedlich. Entsprechend der Bedeutung innerhalb der literarischen Produktion spielt hier die Lyrik nur eine untergeordnete Rolle gegenüber Roman und Drama. Darstellungen der Romantheorie werden - dem methodischen Ansatz von Zola entsprechend - weitgehend auch zu ästhetischen Grundsatzerklärungen. Die Theorie des Dramas wird in ihrem zentralen Aspekt in die Nähe der Theoriereflexion epischer Gestaltung und damit zu grundsätzlichen Abbildproblemen gerückt: "Episches Drama" ist das Stichwort, unter dem das naturalistische Drama poetologisch definiert, aber auch kritisiert wird.

Die theoretisch-kritische Auseinandersetzung um den naturalistischen Roman, die diesen Gattungsteil einleitet, setzt in Deutschland an bei einer Kritik der Zolaschen "analytischinduktiven « (Kapff-Essenther) Methode, Poesie mit Wissenschaft gleichzusetzen. Demgegen- 
über steht die Forderung der frühnaturalistischen Kritiker, mit dem Ton des nationalen Pathos' vorgetragen, nach einem realistischen Roman, der ein umfassendes Zeitbild entwirft. Um die Erschließung also der ganzen Breite modernen Lebens als Romansujet geht es in den Beiträgen der Harts, Irma von Troll-Borostyanis und Franziska von Kapff-Essenthers, ebenso aber um Abgrenzung von der einseitigen Darstellung des Pathologischen, Kriminellen und der sozialen Randmilieus, wie dies offenbar für eine als naturalistisch geltende Modeschriftstellerei dieser Jahre typisch geworden war. Dieser Kritik fällt freilich auch Zola als der vermeintliche Urheber dieser Richtung zum Opfer.

Die "großen Probleme unserer Zeit literarisch [...] fassen " (Bleibtreu), diese Forderung kristallisiert sich immer mehr zur zentralen These naturalistischer Literaturprogrammatik, für deren Umsetzung der Roman als das geeignetste Darstellungsmedium erscheint. Dabei wird aber auch deutlich, daß die theoretischen Begründungen dafür durchaus heterogen, ja oft kaum vermittelbar nebeneinander stehen (Beispiel: Bleibtreu, Bölsche). Die Erschließung neuer Stoffbereiche, die Erweiterung der künstlerischen Gestaltung in Randbereiche der sozialen und psychischen Lebenswirklichkeit hinein wird als die entscheidende historische Leistung naturalistischer Kunstdoktrin, insbesondere für den Roman, angesehen (Litzmann).

Im Rahmen des naturalistischen Romanschaffens spielt der Berlin-Roman eine herausragende Rolle, ja er kann fast als spezifisch naturalistisches Romangenre gelten. Von vorbildhafter Bedeutung für diese Entwicklung in Deutschland waren sicherlich Zolas Paris-Romane, denen ja auch Michael Georg Conrad eine München-Adaption nachschrieb. Im Großstadt-Roman, und hier bot sich in der deutschen Situation lediglich Berlin als Sujet an, konnte der allgemeinen Forderung nach Gestaltung modernen Lebens in seiner avanciertesten Form und im ganzen Spektrum seiner Probleme entsprochen werden. Hinzu kommt, daß der einzige aus der Durchschnittlichkeit naturalistischer Romanproduktion sich abhebende und deswegen vielfach besprochene Roman Max Kretzers, Meister Timpe, ein Berlin-Roman ist.

Drama und Theater des Naturalismus stehen zunächst in durchaus ambivalentem Verhältnis zur naturalistischen Kunsttheorie. Einerseits scheint gerade das Theater die Möglichkeit zu besonders authentischer Wirklichkeitsdarstellung zu bieten, vor allem in der Form einer konsequent illusionistischen Inszenierung, die den Zuschauer in die Haltung des distanziert betrachtenden "Experimentators « versetzt, - andererseits gerät das Drama in seiner konventionellen Bauform in Konflikt mit der Forderung nach Darstellung der Komplexität modernen Lebens und mit der naturalistischen Auffassung vom Menschen als eines von Milieu und Vererbung determinierten, mithin als dramatischer Handlungsträger ungeeigneten Wesens. Solchen Vorstellungen entsprachen weit mehr die epischen Darstellungsmittel. So setzt die Diskussion über "Wesen und Zweck des Theaters" bereits zu Beginn der 80er Jahre ein und begleitet die gesamte Periode. Am Anfang, anlaßgebunden durch den Plan einer Theaterneugründung in Berlin, verbindet sich mit der Forderung nach einem modernen Gegenwartsdrama noch die Vorstellung einer Erneuerung der Nationaltheateridee, im Zusammenhang der für den Frühnaturalismus charakteristischen nationalen Erneuerungspostulate (Heinrich und Julius Hart). Das moderne Drama sollte zugleich ein nationales sein. Diese Vorstellungen 
sind Ende der 80er Jahre zwar abgeschwächt aber noch immer ein Aspekt der Theaterdiskussion. Verstärkt setzt zu dieser Zeit allerdings die Auseinandersetzung mit Dramaturgieproblemen ein als Suche nach einer naturalistischen Form des Dramas. Die Gründung der Freien Bühne Berlin 1889 bedeutet für das naturalistische Drama und Theater einen entscheidenden Durchbruch in der Öffentlichkeit. "Das moderne Theater wird naturalistisch sein - oder es wird gar nicht sein «, erklärt lapidar Otto Brahm, dessen Arbeit als Dramaturg, Theaterleiter und Kritiker der Theaternaturalismus wesentliche Impulse verdankt. Entscheidend für diese Entwicklung ist vor allem auch die Freundschaft und Zusammenarbeit Brahms mit Gerhart Hauptmann. Neben diesem wird Arno Holz zum wichtigsten Neuerer des naturalistischen Dramas; weniger durch seine zusammen mit Johannes Schlaf verfaßten experimentellen Stücke als vielmehr aufgrund seiner sprachkritischen Forderungen: "Sprache des Lebens« als die Sprachform des neuen Dramas. Um "naturgetreue penible Reproduktion der Wirklichkeit« (Paul Ernst) geht es.

Der Durchbruch des naturalistischen Dramas auf der Bühne brachte die Diskussion um eine moderne Dramaturgie (Holz, Schlaf, Spielhagen) in Gang und verschärfte die konservative Kritik erheblich. Der Naturalismus wurde nun in einer eklatanten Form öffentlich, nämlich über die als "moralische Anstalt" hochgehaltene Theaterinstitution. Als das Deutsche Theater Berlin 1894 Hauptmanns Weber aufführte, kündigte Wilhelm II die königliche Loge und strich die staatliche Subvention des Theaters. Beispiele für diese Verschärfung der Naturalismuskritik sind die Beiträge von Gustav Schwarzkopf und Richard Dehmel, die in ihrer Polemik aber zugleich wesentliche Strukturmerkmale der "neuen deutschen Alltagstragödie" (Dehmel) aufzeigen.

Ein eigenes Kapitel widmet die Dokumentation der Freien Bühne Berlin, der Freien Volksbühne Berlin und den Auseinandersetzungen des naturalistischen Theaters mit der Zensur. Gerade in diesem Problemfeld wird der allgemeinpolitische Wirkungszusammenhang erkennbar, in dem der Naturalismus in den frühen 90er Jahren steht. Die Beiträge von Otto Brahm referieren und kommentieren die Geschichte der beiden Theatervereine; wobei die Freie Volksbühne als kulturpolitische Einrichtung der Arbeiterbewegung noch weit mehr im Kreuzfeuer konservativer Kritik steht als der bürgerliche Theaterverein nach Pariser Vorbild. In der Darstellung von Heinrich Bulthaupt werden mit aller Eindeutigkeit die Feindbildstereotypen benannt, unter denen die Konservativen und die Ordnungsbehörden den Naturalismus insgesamt bewerten: als »Kunst des Anarchismus«. Die Darstellungen von Richard Grelling und Paul Schlenther erörtern die "Maßregelungen" (Grelling) der Freien Volksbühne durch Zensurbehörden und Gerichte. Die Verschärfung des politischen Kampfes gegen die Sozialdemokratie, zugespitzt durch die Diskussion der Umsturzvorlage (Dezember 1894 bis Januar 1895) schlug voll durch auf den der sozialdemokratischen Parteigängerschaft bezichtigten Theaternaturalismus.

Die Lyrik ist die im ganzen der Entwicklung des Naturalismus wohl am unprofiliertesten gebliebene Literaturgattung und dies, obwohl die Anthologie Moderne Dichter-Charaktere (1885) als erstes programmatisches Literaturzeugnis die Bewegung in Deutschland öffentlich 
vorstellt. "Unser Credo", so lautet die bekennerhaft überschriebene Einleitung dazu, von Hermann Conradi verfaßt, einem der frühesten Wortführer des Naturalismus. "Wahr und groß, intim und confessionell" sollte die neue Lyrik sein. Gleichermaßen weitgespannte Erwartungen formuliert Karl Henckell; um die "Poesie der Zukunft" geht es, um "große Poesie" schlechthin. 1890 wird von Julius Hart ein erstes Resumee gezogen. Wenn auch eine eigene künstlerische Formensprache hier noch nicht gefunden wird, so hat die naturalistische Lyrik nun doch ein spezifisches Themenrepertoire entwickelt, die Gestaltung der Beziehungen des Menschen "von Klasse zu Klasse». Hart betont ausdrücklich auch die Anklänge an die Revolutionsdichtung der Vormärzzeit. Das Buch Revolution der Lyrik (1899) von Arno Holz ist eine Art Nachruf auf den Naturalismus und weist deutlich schon auf künftige Entwicklungen hin: "Man revolutioniert eine Kunst also nur, indem man ihre Mittel revolutioniert." Mit dieser konsequenten Schlußfolgerung aus seiner bereits 1891 vorgelegten Kunsttheorie sind wesentliche Grundpositionen des Naturalismus, nämlich sein thematischer Zeit- und Gesellschaftsbezug, aufgegeben. Das Formexperiment tritt in den Mittelpunkt.

Insgesamt zeichnet das Gattungskapitel den großen Bogen der Entwicklung einer naturalistischen Formensprache nach und dokumentiert, an die Thesen und Forderungen des Grundlagenkapitels anschließend, die naturalistische Literaturprogrammatik in ihrer gattungsspezifischen Brechung, zeigt ebenso aber die Erweiterung, Modifikation, ja tendentielle Auflösung der konventionellen Gattungsschemata als Folge der kunsttheoretischen Prämissen.

Der dritte Teil der Dokumentation (III) ist der zeitgenössischen Rezeption des Naturalismus vorbehalten. Alle Themen und Problemfelder, die im ersten (Grundlagen) und im zweiten Teil (Gattungen) behandelt wurden, erscheinen hier erneut, in spezifische Diskussionszusammenhänge gestellt und aus parteilicher Sicht bewertet: Naturalismusdiskussion im Spannungsfeld der politischen und literaturkritischen Auseinandersetzungen der Zeit. Die hier zusammengestellten Dokumente sind zu ergänzen durch Texte aus den vorangegangenen Kapiteln, soweit diese aus Rezeptionspositionen heraus geschrieben sind, z. B. die Auseinandersetzungen um den naturalistischen Roman, um Drama und Theater (vgl. dazu die jeweiligen Kommentare).

In einer ersten Gruppe sind Texte zusammengefaßt, die den Naturalismus als Zeitsymptom (»Ausdruck unserer Zeit «) interpretieren, die Abgrenzungen und historische Standortbestimmungen formulieren. So stellt Leopold von Sacher-Masoch den Naturalismus seiner vermeintlichen prinzipiellen Tendenzhaftigkeit wegen als Kunstrichtung generell in Frage: "Eine kranke Literatur für ein krankes Publikum«. Ähnlich argumentiert Karl Frenzel, der auf den Zusammenhang von Naturalismus und Sozialdemokratie hinweist; »Umsturz« im Politischen wie im Ästhetischen ist für ihn der gemeinsame Nenner. Der Naturalismus erscheint Frenzel deswegen als Reflex einer dekadenten Gesellschaft, als Erzeugnis einer gährenden Zeit. Um ein ausgewogenes Urteil ist der liberale Kritiker Max Lorenz bemüht, der die Verdienste der naturalistischen Periode in einer "Erneuerung des künstlerischen Stoffes « sieht, den Naturalismus aber insgesamt - 1899 bereits aus der Erfahrung seiner Auflösung in eine Vielzahl neuer Richtungen und Positionen - als »Übergangsphänomen« bewertet. 
Gleichermaßen um Positionsbestimmungen geht es in den Texten von Wolfgang Kirchbach, der den naturalistischen Autor dem Typus des "sentimentalischen Idealisten « zuordnet und auf das konstruktive Moment (Theorieorientierung) naturalistischer Realitätsauffassung hinweist, und von Maximilian Harden (»In Decadence-Zeiten blüht die Technik immer zu kaum geahnter Höhe empor «). Ernst v. Wolzogen stellt dem naturalistischen »Gesichtswinkel der Bestialität " die künstlerische Sichtweise des »Humors" gegenüber; vor allem aber zieht Wolzogen Verbindungslinien vom Naturalismus zu den vorausgegangenen Realismusbewegungen des 19. Jahrhunderts. Ebenso bemühen sich Moritz Carriere, Friedrich M. Fels und Christian Ehrenfels um Standortbestimmungen des Naturalismus als Kunstrichtung wie als Weltanschauung. Das ganze Spektrum solcher Standortdiskussionen repräsentiert eine Umfrage, die Kurt Grottewitz im Magazin für Litteratur (des In- und Auslandes) 1892 veranstaltet, und in der Vertreter aller weltanschaulichen und literarischen Richtungen zu Wort kommen. "Die Zukunft der deutschen Literatur im Urteil unserer Dichter und Denker" ist die Frage dieser Enquête und zugleich die Perspektive, unter der hier der Naturalismus beurteilt wird.

Als Gruppe für sich sind in dieser Diskussion Dokumente ausgegliedert, die den Naturalismus vom Standpunkt nationalkonservativer, ideologisch sicherlich als präfaschistisch einzuschätzender Sicht diffamieren und sich durch ihren Gestus rigoroser Polemik von anderen Formen zeitgenössischer literarischer Kritik durchaus unterscheiden. Die Texte von Erwin Bauer und Fritz Lienhardt machen heutige Leser betroffen durch die Radikalität, in der hier militanter Antisemitismus und antidemokratische Gesinnung Literaturkritik zum Instrument national-chauvinistischer Agitation werden lassen. Eine Literatur des Deutschtums und der "Rasse-Eigenthümlichkeiten " wird als kulturpolitische Forderung den »internationalen und jüdischen Kopfräsonneurs", den »Berliner Geschäftssemiten Otto Brahm, S. Fischer und Genossen « entgegengehalten. Wie auch in einigen Dokumenten zu den Verbotsprozessen naturalistischer Literatur, insbesondere in Stellungnahmen zu Gerhart Hauptmanns Stück Die Weber, wird hier in aller Deutlichkeit erkennbar, wie die zeitgenössiche Naturalismusdiskussion weit über den internen Diskussionsrahmen von Literatur- und Kunstkritik hinaus zu einer weltanschaulichen und politischen Auseinandersetzung wurde, in der erstmals in aller Eindeutigkeit jenes Diffamierungsvokabular faschistischer Literatur- und Kunstkritik zur Anwendung kommt, das 40 Jahre später den faschistischen Kulturkampf bestimmen sollte.

Anschließend an das Kapitel „Über den Naturalismus hinaus« im Rahmen der Dokumentation der kunsttheoretischen Programatik (Teil I) werden im Zusammenhang der Rezeptionsproblematik weitere Texte vorgelegt, die Naturalismuskritik verbinden mit dem Aufzeigen weiterführender Entwicklungen und um "Gesamtwürdigung « bemüht sind (Bahr, Bartels, Lublinski).

Einen Zusammenhang besonderer Art bilden die Dokumente, die unter dem zeitgenössischen Titel Naturalismus vor Gericht zusammengefaßt sind, die Texte von Conrad Alberti, Julius Röhr, anonyme Dokumete im Zusammenhang mit Zensurprozessen, schließlich Richard Grellings (Gerhart Hauptmanns Anwalt) Glossen zum Weberprozeß. Diese Texte 
reflektieren über die konkreten Rechtsfragen hinaus, aus der Perspektive der allgemeinen Zensurverhältnisse der Zeit, noch einmal das gesamte Bewertungsspektrum, dem der Naturalismus ausgesetzt war. Dabei ist für die Einschätzung durch die Obrigkeitsvertreter entscheidend, daß in die Erörterung des vermeintlichen Rechtsbruchs im Einzelfalle stets die gesamte "neue Richtung « einbezogen und inkriminiert wird. Deutlich wird in der zugespitzten Konfliktsituation der Zensurprozesse aber auch die widersprüchliche Haltung der Naturalisten selbst (Hauptmann) und ihrer Anhänger (Grelling) hinsichtlich konkreter politischer Parteinahmen. Diese Problematik führt unmittelbar in den Problemkreis der Auseinandersetzung der Sozialdemokratie mit dem Naturalismus. Dieser Diskussion wird in der vorliegenden Dokumentation deswegen eine besondere Stellung innerhalb der Rezeptionsdarstellung eingeräumt, weil hier wesentliche Aspekte naturalistischen Selbstverständnisses (Engagement für den Sozialismus und die Arbeiterbewegung, Darstellung des Menschen in seinen sozialen Bezügen, Thematisierung der sozialen Frage) in die kritische Befragung und unmittelbare Konfrontation mit den politisch "zuständigen" Repräsentanten dieser Ideen geraten. Weiterhin wird an diesen Kontroversen erkennbar, wie inhaltlich heterogen im Grunde zentrale Begriffe naturalistischer Programmatik sind und wie schwierig offenbar eine politische Standortfindung für die oppositionelle literarische Intelligenz in den 80er und 90er Jahren des 19. Jahrhunderts war. Zudem konturiert sich in diesen Debatten eine in der Folgezeit zunehmend wichtiger werdende kunsttheoretische Problematik, nämlich die Stellung des Proletariats zur Kunst und die Formulierung von Bedingungen und Zielsetzung einer proletarischen Ästhetik (Franz Mehring). Die Naturalismuskontroverse zeigt, in wie hohem Maße die dabei entwikkelten Stellungnahmen abhängig sind von der allgemeinen politischen und kulturkritischen Theoriereflexion innerhalb der linken Gruppierungen.

In den Texten von Paul Ernst, Wilhelm Liebknecht und Robert Schweichel wird erstmals von sozialdemokratischer Seite aus eine Fundamentalkritik gegen die literarische Moderne formuliert, offenbar als Antwort auf Naturalismus-Sympathisanten im linksoppositionellen Lager der eigenen Partei (Berliner Volkstribüne). Zu diesen Äußerungen nehmen die naturalistischen Autoren unmittelbar Stellung: Brahm, Conrad und Julius Hart antworten auf den "sozialdemokratischen Angriff auf das >jüngste Deutschland`“ (Hart). Die Beiträge von Franz Mehring zum Naturalismus nehmen eine Sonderstellung ein; dazu gehören auch Mehrings Essays zu Ibsen, Zola und Hauptmann, die an anderer Stelle dieses Rezeptionskapitels abgedruckt sind. Es sind eben dies die Beiträge, in denen sich eine programmatische Abgrenzung von bürgerlicher und proletarischer Ästhetik abzeichnet. Ebenfalls einen Block für sich bilden die Dokumente zur Naturalismus-Diskussion der Sozialdemokratie während ihres Parteitags 1896 in Gotha, bei dem es vor allem um die Frage geht, inwieweit der Naturalismus den Interessen einer proletarischen Leserschaft entgegenkommt. In den Zusammenhang dieser Debatte gehören auch die Beiträge von Edgar Steiger, der zu den sozialdemokratischen Anhängern des Naturalismus zählt und der in seiner Funktion als Redakteur der Neuen Welt, einer Unterhaltungsbeilage sozialdemokratischer Zeitungen, im Mittelpunkt der Attacken des Parteitags stand. 
Um 1880 und besonders um 1890 erscheinen die Stücke von Henrik Ibsen auf den Spielplänen der deutschen Bühnen, insbesondere der Berliner. Ibsens dramatisches Werk macht die deutsche Öffentlichkeit mit der literarischen Moderne bekannt, leitet die Diskussion um diese Bewegung mit ein. Der Norweger gilt den Konservativen wie den Sozialdemokraten als "pessimistischer Realist " (Paul Ernst); den Pessimismusvorwurf wiederholt auch noch im Jahre 1900 Franz Mehring in seinem großen Ibsen-Essay. Der jungen Opposition dagegen ist Ibsen das große Vorbild, bringt er doch in seinen Stücken das auf die Bühne, was die Jüngstdeutschen für eine neue Literatur nachdrücklich fordern: Zeitfragen, vor allem die schonungslose Enthüllung der moralischen Korruption der bürgerlichen Gesellschaft. Wie kein anderes literarisches Werk der Zeit wird Ibsens Nora zum Kristallisationspunkt der Diskussion um die Problematik der Frauenemanzipation. Zur Popularität Ibsens in Deutschland trägt sicherlich bei, daß der norwegische Dichter mehrere Jahre auch in Deutschland lebte, in Dresden und in München. Sein Werk ist in diesen Jahren auf den deutschen Bühnen populärer als in seiner norwegischen Heimat.

Die Texte spiegeln das Spektrum der Meinungen und Kontroversen wider, die die IbsenAuseinandersetzung in Deutschland kennzeichnet. Die Beiträge von Otto Brahm und Hermann Bahr argumentieren von Positionen aus, die die positive Bedeutung Ibsens herausarbeiten; Brahm, aus der Haltung großer Bewunderung, weist auf die formalen Neuerungen hin, die Ibsen für die moderne Theaterliteratur gebracht hat; Bahr, mehr um kritische Würdigung bemüht, sieht in Ibsen weniger den Vollender der literarischen Moderne als vielmehr deren Ankündiger. Auch für Emil Reich ist Ibsen, neben Wagner und Nietzsche, der "Prophet dieser neuen Zeit «. Das Feld der Ibsen-Kritiker dagegen ist in sich äußerst heterogen. Dem Sozialdemokraten Paul Ernst gilt Ibsen als Pessimist und damit als Vertreter einer weltanschaulichen Haltung, die dem proletarisch klassenkämpferischen Optimismus zutiefst widerspricht. Für Max Nordau ist der "Ibsenianismus « mit seiner vermeintlichen "Ichsüchtigkeit " Syndrom einer Verfallskultur. Laura Marholm wiederum verweigert der emanzipatorischen aufklärerischen Komponente in Ibsens Werk ihre Zustimmung. Dagegen propagiert sie einen neuen Weibmythos, der sich erklärtermaßen in einen Gegensatz zu Ibsens »Programm der Emanzipationsdamen" stellt.

Im Kreuzfeuer konservativer Naturalismuskritik steht Emile Zola von Anfang an und das aus vielfachen Gründen. Deutsch-nationalem Kulturchauvinismus mußte gerade der Franzose Zola als Inbegriff des Undeutschen (Langbehn) erscheinen. Mit dem Schlagwort vom "Zolaismus « (dem bald auch der Begriff »Ibsenianismus « nachgebildet wurde) faßt die konservative Kulturkritik alle Negativmomente des Naturalismus zusammen; insbesondere auch den internationalistischen Aspekt, der die naturalistische Bewegung auszeichnet, der aus konservativer Sicht freilich als Kulturverrat gebrandmarkt wird. Es sind vor allem zwei Angriffsziele, die Zola bietet: einmal die Thematisierung asozialen Milieus (Verbrechen, Prostitution) und die Schilderung pathologischen psychischen und physischen Verhaltens (Alkoholismus, Wahnsinn); zum andern Zolas theoretisches Programm, das Literatur der Wissenschaft gleichsetzt, verbunden damit die dokumentaristische Methode. Gerade darin sieht die konser- 
vative Kritik ihr traditionelles Kunstverständnis fundamental in Frage gestellt. Das Verwissenschaftlichungspostulat Zolas ist freilich ein Punkt, an welchem auch die deutschen Naturalisten Zola nicht bedingungslos folgen. Auch wenn dessen Schrift Der Experimentalroman als eine der wichtigsten theoretischen Grundlagen der oppositionellen Moderne in Deutschland gilt, wird die Gleichsetzung von Kunst und Wissenschaft nicht nachvollzogen. Hier nehmen die verschiedenen Naturalistengruppen durchaus unterschiedliche Positionen ein. Für die sozialdemokratischen Kritiker, die sich verhältnismäßig spät erst in die Zola-Diskussion einschalten, ist Zola vor allem der Autor des Germinal, des bedeutendsten sozialen Romans der Zeit mit eindeutig politischer Aussage. Das sozialdemokratische Zola-Bild ist deswegen insgesamt eher positiv; Franz Mehring schreibt in seinem Nachruf (1902): "Zola stand turmhoch über seinem kleinen Nachwuchs in Deutschland «. Die Dokumentation versucht das Spektrum dieses vielschichtigen Rezeptionsvorgangs wiederzugeben. Die Texte von Ludwig Pfau, den Brüdern Hart und von Eugen Wolff formulieren die kunsttheoretischen Vorbehalte gegen Zolas Experimental- bzw. Dokumetar-Ästhetik. Diese sei, so resümiert Wolff, der "Tod der Poesie«, und er trägt damit die Grundthese jener Zola-Kritiker vor, die sich in ihrer Auseinandersetzung argumentativ auf den kunsttheoretischen Ansatz des Franzosen einlassen, diesem freilich ihre andere Position entgegenhalten. Eine Gruppe militanter Zola-Gegner wird erkennbar in den Beiträgen von Amyntor, Langbehn und auch von Fritz Mauthner. Diese Autoren verurteilen Zola vom moralischen Standpunkt aus rigoros, sie tragen jene Anschuldigungen vor, die beinahe stereotyp gegen die gesamte Moderne vorgebracht werden: Pornographie, Schmutzliteratur und opportunistische Geschäftemacherei mit der literarischen Sensation, Volksverderber. Selbst so differenziert argumentierende Kritiker wie Maximilian Harden sprechen Zola, den er als den "Schlachtenmaler des animalischen Lebens" apostrophiert, aus moralischem Vorbehalt jedwede Künstlerschaft ab.

Für Michael Georg Conrad dagegen, den Herausgeber der Gesellschaft, ist Zola der »Großmeister des Naturalismus«. Conrads Beiträge sind aus einer Haltung ergebener Parteigängerschaft, ja fast schon der Huldigung geschrieben. Um eine positive Würdigung Zolas ist auch Hermann Bahr bemüht, obwohl sich für ihn doch deutlich die Begrenztheit von dessen Ästhetik abzeichnet und er bald der ganzen naturalistischen Bewegung absagen wird. Der Zola- Essay von Georg Brandes stellt in diesem Rahmen die wohl substantiellste und umfassendste Charakteristik Zolas als Künstler dar. Diesen rundum positiven Zola-Bildern schließen sich mit gleicher Tendenz die Beiträge von Lafargue und Franz Mehring an, die beide in der sozialdemokratischen Zeitschrift Die neue Zeit erschienen sind; für beide Autoren ist Zola der einzige moderne Autor, der den Menschen in der ganzen Komplexität seiner sozialen Bindungen und Zwänge gestaltet: "Schilderung und Analyse der ökonomischen Riesenorganismen der Neuzeit « (Lafargue).

Texte zur Dokumentation der Rezeption von Gerhart Hauptmanns Stück Die Weber schließen den Rezeptionsteil ab. Die Weber waren ohne Zweifel das die zeitgenössische Öffentlichkeit provozierendste Werk des naturalistischen Theaters - ja darüber hinaus wohl auch der gesamten naturalistischen Bewegung in Deutschland. In vieler Hinsicht stellt dieses 
Stück einen Ausnahmefall dar, gleichwohl bündeln sich hier alle Linien naturalistischer Grundsatzdiskussionen. In einer kaum übersehbaren Serie von Zensurprozessen werden alle Argumente für und gegen den Naturalismus erneut diskutiert; es ist eine Diskussion, die immer wieder auch die grundsätzliche Frage der Kunst- und Meinungsfreiheit in den Mittelpunkt rückt.

Ausgewählt wurden Texte, die die Buchausgabe der Weber (1892), die Aufführung an der "Freien Bühne Berlin« (1893) und schließlich am »Deutschen Theater" zu Berlin (1894) besprechen. Wie an keinem anderen Werk des Naturalismus gerät am Beispiel der Weber das ästhetische Programm des Naturalismus in die kritische Befragung nach dem sozialen Sinn von Literatur, ihrer Stellung zu den "großen Fragen der Zeit«. Diesen Fragen sich in aller Konsequenz zu stellen, war das Programm mit dem der Naturalismus angetreten war.

III

Die Arbeitsteilung der Herausgeber gestaltete sich so, daß die Kommentierung der Dokumente von Christine Müller erarbeitet wurde. Es sind Sachkommentare, vor allem aber Einführungen in die Problemzusammenhänge, in denen die einzelnen Texte stehen. Um den Kommentar zu entlasten, sind Daten zu Werken und Personen, die über die üblichen Nachschlagewerke leicht zugänglich sind, nicht aufgenommen. Eingehend erläutert werden dagegen die zum Verständnis der Texte erforderlichen kunstprogrammatischen und weltanschaulichen Standpunkte der Autoren, ein Aspekt, der insbesondere für die Analyse der Rezeptionsproblematik von Bedeutung ist, ebenso aber auch für die Differenzierung der Positionen innerhalb der Gruppen der naturalistischen Autoren. In der Regel ist deswegen auch über den jeweiligen Text hinaus Bezug genommen auf das Problemumfeld, auf Positionen und Diskussionen, die nicht in die Dokumentation aufgenommen wurden, jedoch in den Kontext der Auseinandersetzung gehören. Die dem Dokumententeil angeschlosssene Bibliographie verzeichnet die einschlägige Forschungsliteratur und ergänzende Quellensammlungen.

Die editorische Gestaltung der Texte entspricht den Gepflogenheiten der Reihe der Manifeste. Die Texte wurden möglichst in ihrer originalen Form belassen. Orthographische Fehler oder offensichtliche Druckfehler wurden stillschweigend korrigiert. Die Interpunktion der Vorlage wurde übernommen, Hervorhebungen durch Sperrdruck, Kursivsatz oder Fettdruck sind einheitlich kursiv gesetzt. Kursiv erscheinen außerdem alle Werktitel und die Namen von Zeitschriften, Jahrbüchern etc. Der genaue bibliographische Nachweis ist dem jeweiligen Text vorangestellt. In der Regel wurde der Erstdruck als Druckvorlage herangezogen.

Das für die Dokumentation durchgesehene Material ist ein Vielfaches von dem, was letztendlich in den Band aufgenommen werden konnte. In Ermangelung bibliographischer Hilfsmittel für diesen Bereich (eine Naturalismusbibliographie bleibt weiterhin ein Desiderat der historischen Forschung), mußte systematisch recherchiert werden; die Durchsicht von Zeitschriften erhielt dabei ein besonderes Gewicht, entsprechend der Absicht, auch die zeitge- 
nössische Rezeption des Naturalismus, die sich weitgehend in der Zeitschriftenpublizistik niederschlug, in die Dokumentation mit einzuarbeiten. Tageszeitungen wurden nur auf spezifische Diskussionen begrenzt einbezogen. Für die Beschaffung des vielfältigen, oft entlegenen Materials ist vor allem der Hamburger Staats- und Universitätsbibliothek zu danken, für Teilbereiche auch der Staatsbibliothek Berlin.

Besonderer Dank gebührt Dr. Bernd Lutz, der dieses Buch als Lektor mit großem Engagement betreut hat und über Jahre hin ein geduldiger Gesprächspartner war.

Hamburg, im Juni 1987

Manfred Brauneck

1 P. Bürger: Naturalismus - Ästhetizismus und das Problem der Subjektivität. In: Naturalismus/Ästhetizismus. Hrsg. v. Ch. Bürger, P. Bürger, J. Schulte-Sasse. Frankfurt 1979, S. 21

2 Vgl. Geschichte der deutschen Literatur von 1830 bis zum Ausgang des 19. Jahrhunderts. Bd. 8, 2 (von einem Autorenkollektiv, Leitung u. Gesamtbearbeitung K. Böttcher zs. mit P. G. Krohn u. P. Wruck) Berlin (Ost) 1975, S. 1005; insbesondere auch der Hinweis auf die Geschichte der deutschen Literatur. Hrsg. v. Institut für Weltliteratur A. M. Gorki der Akademie der Wissenschaften der UdSSR. Bd. 4 (Red. R. M. Samarin u. J. M. Fradkin). Moskau 1968

3 Vgl. R. Brinkmann: Expressionismus. Forschungs-Probleme. 1952-1960. Stuttgart 1961

4 D. Borchmeyer: Der Naturalismus und seine Ausläufer. In: Geschichte der deutschen Literatur vom 18. Jahrhundert bis zur Gegenwart. Hrsg. v. V. Žmegač. Bd. II,1. Königstein/Ts. 1980, S. 158.

5 Vgl. M. Brauneck: Literatur und Öffentlichkeit im ausgehenden 19. Jahrbundert. Studien zur Rezeption des naturalistischen Theaters in Deutschland. Stuttgart 1974, S. $20 \mathrm{ff.}$ 